\title{
A new method to determine anastomosis angle configuration for arteriovenous fistula maturation
}

\author{
Mohammad Rezapour ${ }^{1}$, Mohammad Mehdi Sepehri ${ }^{2}$, Morteza Khavanin Zadeh*3, Mahmood Alborzi ${ }^{4}$
}

Received: 5 Sep 2016

Published: 25 July 2018

\begin{abstract}
Background: The kidneys of patients with chronic kidney disease (CKD) do not function well enough and those in end-stage renal disease (ESRD) of CKD need hemodialysis (HD) as a common renal replacement therapy (RRT) procedure. HD requires a vascular access (VA), and arteriovenous fistula (AVF) is the common VA choice in the world due to its very few complications. Despite the widespread use of AVFs, some risk factors maximize AVF failure, which is accompanied by complications of the patient such as repeating VA surgeries and hospitalization. Therefore, finding effective factors in the success of surgery is highly important and, thus, this study aimed at measuring the effect of anastomosis angle on the success of AVF surgery.

Methods: This study evaluated the effect of conducted angle in an AVF anastomosis on AVF maturation. The images of 48 created AVFs for CKD patients was provided over a one-year period (from May 2016 to April 2017). Cross-tab analysis was used, and significance level was considered meaningful at $p$-value $\leq 0.001$. A centralized database was designed to integrate data. A method for image processing was developed and geometrical characteristics of the vessels (such as anastomosis angle) and also the diameter of artery and vein were measured via AutoCAD 2017 software and exported to the database along with other data.

Results: The rate of the AVF failure in the studied patients was $8.96 \%$. The anastomosis angle $\leq 30^{\circ}$ is preferable from the AVF status point of view because most AVF maturation (or least AVF failure) rates are detected at this range.

Conclusion: This study was performed based on a new approach without the need to measure hemodynamic parameters. Moreover, it signified the important role of anastomosis angle in the function of AVF, showing that the anastomosis angle $\leq 30^{\circ}$ is a preferable
\end{abstract} intraoperative recommendation for AVF surgery.

Keywords: Vascular access surgery, Hemodialysis, Anastomosis angle, AVF failure, AVF maturation, Surgical process improvement Copyright $₫$ Iran University of Medical Sciences

Cite this article as: Rezapour M, Sepehri MM, Khavanin Zadeh M, Alborzi M. A new method to determine anastomosis angle configuration for arteriovenous fistula maturation. Med J Islam Repub Iran. 2018 (25 July);32:62. https://doi.org/10.14196/mjiri.32.62

\section{Introduction}

End-stage renal disease (ESRD) is the final stage of chronic kidney disease (CKD) in which the kidneys no longer function well enough to meet the needs of daily life (1), and it has been estimated that almost $10 \%$ of the worldwide population is affected by CKD (2). Patients with

Corresponding author: Dr Morteza Khavanin Zadeh, khavaninzadeh.m@iums.ac.ir

1. Department of Information Technology Management, Science and Research Branch, Islamic Azad University, Tehran, Iran.

2. Faculty of Industrial and Systems Engineering, Tarbiat Modares University, Tehran, Iran, \& Hospital Management Research Center, Iran University of Medical Sciences, Tehran, Iran.

3. Faculty of Medicine, Iran University of Medical Sciences, Hasheminejad Kidney Center (HKC), Tehran, Iran.

4. Faculty of IT Management, Science and Research Branch, Islamic Azad University, Tehran, Iran
ESRD need renal replacement therapies (RRTs) such as hemodialysis (HD), peritoneal dialysis (PD), or kidney transplantation (3). Due to some conditions in the us e of RRT (such as the shortage of available kidneys for transplantation), HD is the most common method used in

$\uparrow$ What is "already known" in this topic:

The anastomosis angle of AVF is of great importance for surgeons who are working in this field, as it helps increase the maturation of the fistula. However, most researchers have calculated hemodynamic conditions, specially "wall shear stress", which need measuring the properties of blood flow and internal diameter of the vessel.

\section{$\rightarrow$ What this article adds:}

A new approach was provided to convert intraoperative images of fistulas into quantitative data. There was no need for additional specifications, and the analyses determined the optimal angle, which reduced the failure rate of the fistula operation. Moreover, the anastomosis angle had to be below or equal to 30 degrees $\left(\leq 30^{\circ}\right)$. 
these patients (4).

HD requires an access for connecting the dialyzer machine to the patient's vascular system, and this connection is provided by a vascular access (VA). There are 3 main types of VA: (1) arteriovenous fistula (AVF), (2) arteriovenous graft (AVG), and (3) central venous catheter (CVC). AVF has the least association with morbidity and mortality, thus, different countries strongly recommend AVF (5). AVF remains the "first choice" for chronic HD (5) and the closest to the "ideal model" of VA (6). However, primary failure remains a common problem impeding arteriovenous fistula (AVF) maturation and increasing patients' morbidity and mortality (7). The major cause of AVF failure is either unsuccessful maturation or venous stenosis of a matured AVF (8). Moreover, the quality of the inflow artery and the outflow vein has consistently been associated with AV access success (9). Nevertheless, the rates of inadequate AVF maturation and functional failure are alarmingly high (10).

AVF is created by communication between artery and vein. Evaluating the effects of modificable configurations on the results of VA surgery, such as the conducted angle between vessels, can reduce AVF failure rate and improve AVF maturation. Therefore, finding these factors is highly important for identifying complications of the patient, such as repeating VA surgeries and hospitalization. This study was conducted to provide an image processing method and measure the effect of anastomosis angle on the success of AVF surgery.

\section{Methods}

This study was implemented based on images of AVF surgeries and it calculated the measures of angles between the involved vessels in any AVF. Then, the status of each patient's AVF was followed and the relationship between the angle and AVF maturation / failure was found.

Statistical method was crosstab analysis, and significant level was considered meaningful at $p$-value $\leq 0.001$. A centralized database was designed for integrating variety data. A method for image processing was developed and geometrical characteristics of the of vessels, such as anastomosis angle, and the diameter of artery and vein were measured via AutoCAD 2017 software and exported to database along with other data.

\section{Study variables}

To achieve the goals of the study, the data of 48 ESRD patients were collected in 3 stages: (1) preoperative (age, gender, marital status, weight, height, smoking, diabetes mellitus (DM), hypertensive, hearth disease, drugs, CKD duration), (2) intraoperative (AVF hand (right/left), AVF site (radial/radial up), anastomosis type (end-to-side (ETS) /side-to-side (STS)), image processing and vessels characteristics measuring), and (3) postoperative (AVF status (failure/ maturation)). One of the AVF images is shown in Fig. 1; the vessel is the vein connected to artery (the below vessel):

\section{Inclusion criteria were as follow}

(i) ESRD patients who referred by a nephrologist for
AVF creation from May 2016 to April 2017;

(ii) Range of each patint's BP had to be more than $100 / 60$ and less than $145 / 90$ in day of operation.

\section{Exclusion criteria were as follow}

(i) BP under 100/60;

(ii) Veins with diameter $<2 \mathrm{~mm}$ because they have no suitable vein continuity;

(iii) Artery with atherosclerosis or one that was not suitable due to having diameters $<2 \mathrm{~mm}$;

(iv) Patints who have used anti-coagulation drugs;

(v) Or those whose vascular system was prepared only for brachiocephalic VA, but not for radio-cephalic VA.

In this study, similar to many other studies $(11,12)$, to examine the effects of gathered parameters on thrombosis, the role of this variable was considered as the dependent (label) variable.

The final material was constructed from merging 3 stages of data: (i) demographic data of patients, (ii) images of their created AVF, and (iii) status of their AVF after surgery.

\section{Ethical approval of the research}

All participants gave informed consent before participation. The patients were admitted and underwent native AVF operation by the same surgeon to receive HD. The patients were informed about the study and their written consent was obtained.

\section{Methodology}

The images were imported into AutoCAD 2017 software and the geometrical characteristics of the vessels, such as anastomosis angle creating AVF, and also the diameter of
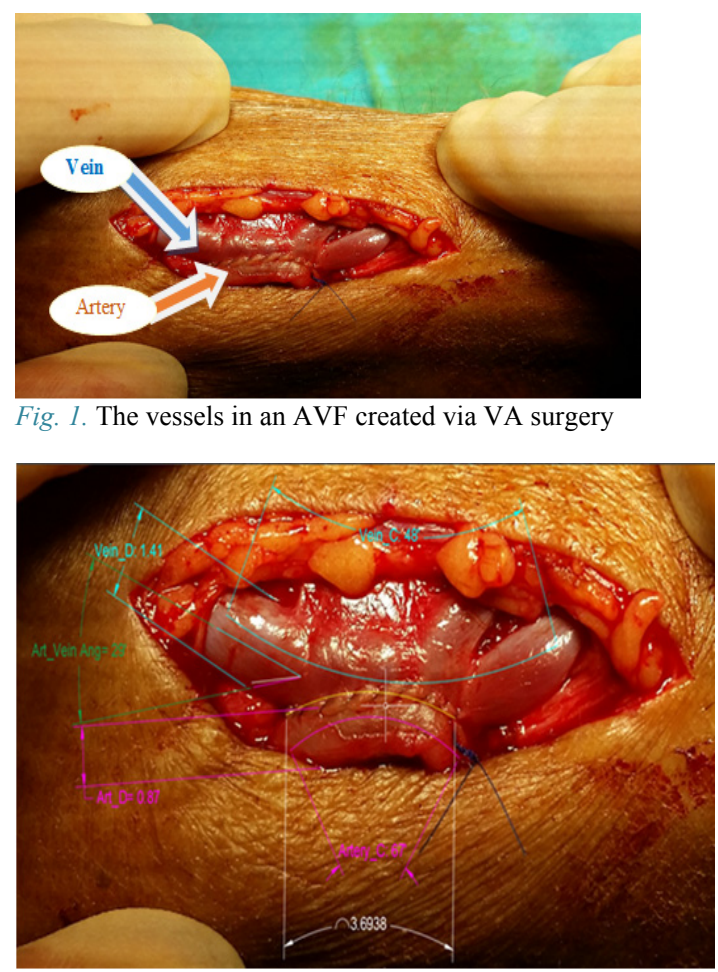

Fig. 2. Calculating measures of AVF parts: The green and yellow colors show the anastomosis angle and length of AVF, respectively. Also, the pink and cyan colors show the vessels diameter and their curve, respectively. 


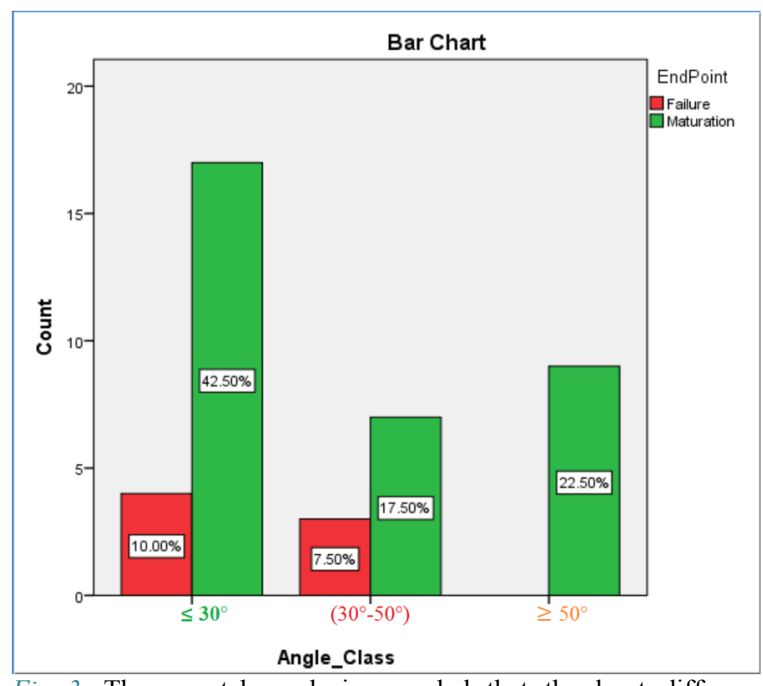

Fig 3. The crosstab analysis revealed that the least difference of AVF maturation and AVF failure rate occurred for anastomosis Angles $\leq 30^{\circ}$.

artery and vein were measured using this software. One of these images, which consists of measures of AVF, is shown in Fig. 2.

\section{Results}

Considering the interpretability of the classified categories and according to the calculated measures of created angles, all categories were classified into 3 parts: (1) less than or equal to $30^{\circ}$, (2) $30^{\circ}-50^{\circ}$, and (3) $\geq 50^{\circ}$.

The results of crosstab analysis confirmed that the anastomosis angle is a major determinant of the hemodynamics factor and that the lowest difference between AVF maturation rate and AVF failure rate exists in degrees $\leq 30^{\circ}$ of anastomosis angle (Figure 3).

Despite the lower rate of AVF failure in the second bar in Figure $3\left(17.5 \%\right.$ in $\left(30^{\circ}-50^{\circ}\right)$ and in the third angle range $\left(\geq 50^{\circ}\right)$, there was no AVF failure. However, the difference of AVF maturation rate and AVF failure rates in them were
$10 \%$ and $22.5 \%$, respectively. Therefore, the most meaningful difference existed in the first angle range $\left(\leq 30^{\circ}\right)$ (maturation rate - failure rate $=\underline{32.5} \%$ ). Thus, it can be concluded the anastomosis angle $\leq 30^{\circ}$ is a preferable intraoperative recommendation for AVF surgery, and selecting this angle range during operation is as an effective factor in the success of the surgery.

\section{Discussion}

\section{AVF Failure and Patency Rates}

The rate of AVF failure varies significantly in the literature, leading to heterogeneity in the published primary patency rates (12).

In a 2014 study, of 338 autologous AVFs, 326 patients had obvious AVFs for dialysis (96.4\% surgical success rate, or $3.6 \%$ AVF failure rate) (13). Sari et al. detected AVF failure in 81 patients out of 831 studied cases (rate = $10 \%$ ) (14), and Monroy-Cuadros et al. reported $15.3 \%$ for incidence rates of primary AVF filure and never using AVF after operation (15).

Predictably, in a meta-analysis on 46 articles $(7,393$ AVFs), the overall risk of primary failure was $23 \%$, but it increased to $37 \%$ in the elderly $(12,16)$, and primary fistula function was achieved in 26 of 43 patients $(60 \%)$, meaning that AVF failure rate was equal to $40 \%$ (17). In the past years, a worse situation has been reported indicating that from 101 fistulas only 47 fistulas (46.5\%) developed sufficiently to be used for dialysis, representing the AVF failure rate of $53.5 \%$ (18).

The rate of AVF failure in this study $(8.96 \%)$ was not very high compared to the average reported AVF failure rates in other parts of the world. We published similar results in 2008 (7) and found the failure rates of $8.05 \%$ at that time; and in an article in 2013 (19), we observed the failure rates of $7.45 \%$ from Dec 2010 to Nov 2010. These reports show that the AVF failure rate did not have any significant changes.

Table 1. Reported rates of AVF failure in researches during 1999 and 2017

\begin{tabular}{lcccc}
\hline Reference. & Publishing Year & Region & No. Patients & AVF Failure rate \\
\hline Miller et al. & 1999 & Birmingham, US & 101 & $53.5 \%$ \\
Tordoir et al. & 2003 & Netherlands & 43 & $40 \%$ \\
Khavanin Zadeh et al. & 2008 & Iran & 100 & $8.05 \%$ \\
Monroy-Cuadros et al. & 2010 & Canada & 831 & $10 \%$ \\
Rezapour et al. & 2013 & Iran & 193 & $7.45 \%$ \\
MacRae et al.; Al-Jaishi, et al. & 2014 & London & 7,393 & $23-37 \%$ \\
Yoo et al. & 2014 & Busan & 338 & $3.6 \%$ \\
Sari et al. & 2016 & Turkey & 36 & $15.3 \%$ \\
Rezapour et al. & 2018 & Iran & 48 & $8.96 \%$ \\
\hline
\end{tabular}

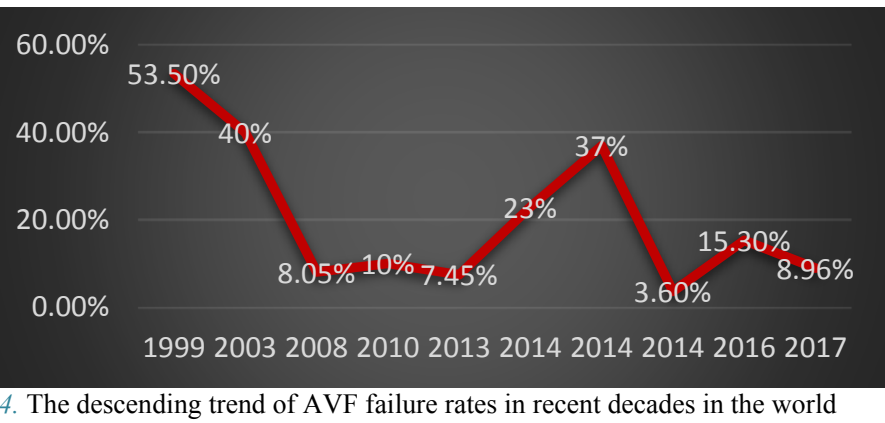


Table 1 summarizes the discussed range of AVF failure rates, sorted according to year of publication.

Figure 4 illustrates the mentioned AVF failure rates, showing a descending trend of AVF failure rates in recent decades

\section{Risk factors that cause AVF failure}

Fistula failure factors are studied using various methods. Parts of studies have focused on discovering influential rules between biomechanical, geometrical, and thermodynamical parameters, such as the effect of similar diameters of vein and artery vessels on the AVF maturation (20). Some other researchers have tried to emphasize the associations between characteristics of ESRD patients (such as positive history of hypertension and/or diabetes mellitus (DM)) with the AVF failure result (19,21-22), which overall can be useful in predicting surgical complications, specially reasons of nonmaturation in AVF after VA operation. Also, when data mining techniques are applied, some "inverse effects" of these characteristics on the AVF are revealed, such as the unusual effect of hemoglobin (HB) level in smoking HD patients (23) or proper behavior of non-hypertensive ESRD patients towards their AVF maturation versus risk of positive history of Blood Pressure (BP) (24-25). Moreover, these studies provide insight that helps constructing a system for VA surgeons (26) to provide them with hints about adjusting preoperative factors of ESRD patients, such as HB level (7,11), DM (19, 27-28), triglyceride and phosphate (29), and BP (30), to increase the likelihood of AVF maturation.

From another perspective, performing a meta-analysis on published researches concludes many inscrutable points in different studying conditions; for example, there are conflicts on risk of BP toward AVF failure. While a study in 2016 on 36 HD patients did not find any relationship between BP and thrombosis formation $(p>0.05)(14)$, in 2 more detailed reviews on $87 \mathrm{HD}$ patients, a significant relationship was found between the systolic blood pressure (SBP) and the delay of AVF maturation (30). Moreover, under publishing research in Nature journal, which was done on 480 HD patients, indicated that the patients with a positive history of hypertension have more matured AVF (31).

Therefore, there are relatively contradictory views about the mentioned non-geometric factors. However, more geometric studies should be conducted to achieve a more accurate and realistic low-risk AVF failure model. In this study, we examined the association between potentially modifiable VA factors, particularly the effect of angle of artery and venous vessels, on the AVF maturation. The results can play a consultative role in practical decision-making for surgeons and can also provide an intraoperative model for vascular access surgery (VAS).

\section{Effects of anastomosis angle on AVF}

The effects of hemodynamics factors on AVF maturation have been studied in some researches in recent years (24). In a 2016 published study, it was found that "maturation time", the time after VA operation and starting HD, decreases when diameters of the vein and artery are close to one another (19). In accordance with Poiseuille's law (32), the radius of a stenosis is much more significant than its length (33-36).

Anastomotic model for AVF operation is studied by other researchers using different methods. Ene-Iordache et al. have employed Wall Shear Stress (WSS) analysis and found that in AVF for HD, the anastomosis angle does affect the local disturbed flow patterns and that acute angles would lead to less intima formation, either on the vein or arterial side. They recommended that clinicians consider this at the time of AVF creation because anastomosis angle is in part amenable to surgical manipulation. Also, they concluded that an acute angle $\left(30^{\circ}-40^{\circ}\right)$ is the preferred choice (37).

More precisely, in chapter 3 of Ene-Iordache's book (38), which is based on his previous studies and those of his colleagues (39), he stated, "We have found that in side-to-end radial-cephalic AVF for HD, the anastomosis angle does impact on the local disturbed flow patterns. Among the 4 geometries $\left(30^{\circ}, 45^{\circ}, 60^{\circ}\right.$ and $\left.90^{\circ}\right)$, the smaller angle $\left(30^{\circ}\right)$ would be the preferred choice that minimizes the development of neointima. Clinicians should consider this at the time of AVF creation because anastomosis angle is in part amenable to surgical manipulation."

Silva J.A et al. have analyzed the blood flow through computational modeling in AVF used in HD (40). They obtained the possibility of stenosis formation caused by hyperplasia by computing the results for WSS, oscillatory shear index (OSI), velocity, and local circulation fields, and they found that higher angles present more secondary flows and larger extensions of stagnation regions near the critical areas of the junctions. According to their mentioned results, "a range around $25^{\circ}$ was identified to be the most suitable choice for clinical applications, minimizing the possibility of diseases."

Similarly, Grus et al. calculated the parameters of the flow field, such as WSS and OSI, and showed that the maximum of spatial gradient of WSS decreased and OSI increased with wider bypass and more acute angle (41). Then, they concluded that more acute anastomosis angle promotes hemodynamics known to reduce formation of intimal hyperplasia.

On the other hand, in a 2012 study on radio cephalic arteriovenous fistula (RCAVF) at wrist, Hassan et al. investigated 18 three-dimensional models of RCAVF and they concluded that for an average flowrate, anastomosis angle should be maintained between $45^{\circ}$ and $60^{\circ}$ to minimize adverse effects (42). Also, a mathematical study on an endto-side (ETS) AVF has investigated the hemodynamic impact of anastomosis size and angle on pressure drop and flow distribution (43). They concluded that pressure drop decreased with a larger anastomosis cross-sectional area and an angle wider than $43^{\circ}$, while it was almost stable for smaller angles. In a 2013 study (44), Jeffery et al. found that venous outflow, which is associated with AVF aneurysm (AVFA) (45), is the lowest by configurations angle of $45^{\circ}$ in ETS anastomosis. 
Table 2. Recommended degrees for anastomosis Angle in AVF operation

\begin{tabular}{lccc}
\hline Reference. & Publishing Year & No. Patients / Models & Analysis Method \\
\hline Ene-Iiordache et al. & $2015-2013$ & 4 & Computational Fluid Dynamic (CFD) \\
Van Canneyt et al. & 2010 & 1 & Mathematical \\
Ene-Iordache et al. & 2012 & 4 & WSS \\
Hassan et al. & 2012 & 18 & CFD \\
Hull et al. & 2013 & 17 & CFD \\
Silva et al. & 2015 & 6 & WSS and OSI \\
Grus et al. & 2016 & 3 & WSS \\
Rezapour et al. & 2018 & 48 & $0^{\circ}-40^{\circ}$ \\
\hline
\end{tabular}

Table 2 presents the recommended degrees for anastomosis angle in AVF operation

Calculating hemodynamic conditions, such as WSS, requires measuring the properties of blood flow and internal diameter of the vessel; for instance, Gnasso et al. have calculated WSS according to the following formula (46):

Wall shear stress $=$ blood viscosity $\times$ blood velocity $/$ internal diameter of vessel.

Which needs measuring the properties of the blood flow and the internal diameter of the vessel. However, the presented method does not need these specifications to reach the desired angle.

\section{Conclusion}

Summing up the rates of this study and other published rates of AVF failure in the world shows that the trend of AVF failure rates has been descending in the recent decades, which is a promising outcome for the treatment of ESRD patients by this VA method.

Vessels analyses showed that the best angle between artery and vein, which has the lowest rate of AVF failure, should be maintained around $30^{\circ}$ during operation. The finding can become an intraoperative pattern to configure angle among AVF surgical vessels.

Although the present studyuse a new method for measuring VAS components, the results of other researches support our conclusion. Therefore, our invented methodology can be used by other researchers.

\section{Acknowledgment}

This article was partly based on the results extracted from Ph.D. thesis currently defended by the first author (Dr. Mohammad Rezapour) at the Department of Information Technology Management, Science and Research Branch, Islamic Azad University, Tehran, IR Iran, supervised by Prof. Mohammad Mehdi Sepehri and Dr. Morteza Khavanin Zadeh (M.D) and advisory of Dr. Mahmood Alborzi.

\section{Conflict of Interests}

The authors declare that they have no competing interests.

\section{References}

1. Kim JU, Kim M, Kim S, Nguyen TT, Kim E, Lee S, Kim S, Kim H. Dendritic Cell Dysfunction in Patients with End-stage Renal Disease. Immune network. 2017 Jun 1;17(3):152-62.

2. Mihajlovic M, Heuvel LP, Hoenderop JG, Jansen J, Wilmer MJ, Westheim AJ, Allebes WA, Stamatialis D, Hilbrands LB, Masereeuw R. Allostimulatory capacity of conditionally immortalized proximal tubule cell lines for bioartificial kidney application. Sci Report. 2017 Aug 2;7(1):7103.

3. Webster AC, Nagler EV, Morton RL, Masson P. Chronic kidney disease. The Lancet. 2017 Mar 25;389(10075):1238-52.

4. Borzou SR, Mahdipour F, Oshvandi K, Salavati M, Alimohammadi N. Effect of mealtime during hemodialysis on patients' complications. J Caring Sci. 2016 Dec;5(4):277.

5. Santoro D, Benedetto F, Mondello P, Pipitò N, Barillà D, Spinelli F, Ricciardi CA, Cernaro V, Buemi M. Vascular access for hemodialysis: current perspectives. Intl J Nephrol Renovascul Dis. 2014;7:281.

6. Stolic R. Most important chronic complications of arteriovenous fistulas for hemodialysis. Medl Principl Pract. 2013;22(3):220-8.

7. Khavanin MZ, Gholipour F, Hadipour R. The effect of hemoglobin level on arteriovenous fistula survival in Iranian hemodialysis patients. J Vascul Access. 2008;9(2):133-6.

8. Zarjou A, Agarwal A. Superoxide in AVF dysfunction: a new target for intervention. Am J Physiol Renal Physiol. 2012 Dec 15;303(12):F1599600.

9. Cronenwett JL, Johnston KW. Rutherford's Vascular Surgery E-Book. Elsevier Health Sciences; 2014 Mar 12. Section 13, Chapter 73: Huber T.S., P. 1087.

10. Vazquez MA. Vascular access for dialysis: recent lessons and new insights. Current Opinion in Nephrology and Hypertension. 2009 Mar $1 ; 18(2): 116-21$

11. Duque JC, Tabbara M, Martinez L, Cardona J, Vazquez-Padron RI, Salman LH. Dialysis arteriovenous fistula failure and angioplasty: intimal hyperplasia and other causes of access failure. AM J Kidney Dis. 2017 Jan 1;69(1):147-51.

12. MacRae JM, Dipchand C, Oliver M, Moist L, Lok C, Clark E, Hiremath S, Kappel J, Kiaii M, Luscombe R, Miller LM. Arteriovenous access failure, stenosis, and thrombosis. Canadian J Kidney Health Dis. 2016 Sep 26;3:2054358116669126.

13. Yoo DW, Yoon M, Jun HJ. Successful Access Rate and Risk Factor of Vascular Access Surgery in Arm for Dialysis. Vascul Special Intl. 2014 Mar;30(1):33

14. Sarı F, Taşkapan H, Sı ğırcı A, Akpınar B. Evaluation of Risk Factors for Arteriovenous Fistula Failure in Patients Undergoing Hemodialysis. Erciyes Med J/Erciyes Tip Dergisi. 2016 Mar 1;38(1).

15. Monroy-Cuadros M, Yilmaz S, Salazar-Bañuelos A, Doig C. Risk factors associated with patency loss of hemodialysis vascular access within 6 months. Clin J Am Soci Nephrol. 2010 Oct 1;5(10):1787-92.

16. Al-Jaishi AA, Oliver MJ, Thomas SM, Lok CE, Zhang JC, Garg AX, Kosa SD, Quinn RR, Moist LM. Patency rates of the arteriovenous fistula for hemodialysis: a systematic review and meta-analysis. Am J Kidney Dis. 2014 Mar 1;63(3):464-78.

17. Tordoir JH, Rooyens P, Dammers R, van der Sande FM, de Haan M, Yo TI. Prospective evaluation of failure modes in autogenous radiocephalic wrist access for haemodialysis. Nephrol Dialysis Transplant. 2003 Feb 1;18(2):378-83

18. Miller PE, Tolwani A, Luscy CP, Deierhoi MH, Bailey R, Redden DT, et al. Predictors of adequacy of arteriovenous fistulas in hemodialysis patients. Kidney Intl. 1999;56(1):275-280.

19. Rezapour M, Khavanin Zadeh M, Sepehri MM. Implementation of predictive data mining techniques for identifying risk factors of early AVF failure in hemodialysis patients. Comput Math Method Med. 2013;2013.

20. Rezapour M, Taran S, Parast MB, Zadeh MK. The impact of vascular diameter ratio on hemodialysis maturation time: Evidence from data mining approaches and thermodynamics law. Med J Islam Repub Iran. 2016;30:359.

21. Sepehri MM, Khavaninzadeh M, Rezapour M, Teimourpour B. A data mining approach to fistula surgery failure analysis in hemodialysis patients. InBiomedical Engineering (ICBME), 2011 18th Iranian Conference. 2011 Dec 14:15-20).

22. Khavanin Zadeh M, Rezapour M, Khavanin Zadeh E, Balin Parast M, Rezapour H. The Relationship between Risk Factors of Hemodialysis 
Patients and Arterio Venus Fistula Maturation at Hasheminezhad Hospital. Iranian J Surg. 2015;22(4):54-64.

23. Khavanin Zadeh M, Rezapour M, Sepehri MM. Data mining performance in identifying the Risk Factors of early arteriovenous fistula failure in Hemodialysis Patients. Intl J Hospital Res. 2013 Mar $1 ; 2(1): 49-54$

24. Wayne EJ, Brier ME, Dwyer AC. Association of maturation period blood pressure with dialysis access patency. In: Seminars in dialysis 2013;26(1):90-96

25. Rezapour M., Sepehri M.M, Khavanin Zadeh M., Alborzi M. Data Mining Application for Detect Impacts of Infection and Hypertension on Vascular Surgery Complications, 2th International Conference on Knowledge-Based Research in Computer Engineering \& Information Technology, 2017.

26. Rezapour M, Sepehri MM, Khavanin Zadeh M, Alborzi M. Forecasting Surgical Outcomes Using a Fuzzy-Based Decision System". Intl J Hospital Res. 2017;6(3).

27. Savaj S, Abdi E, Nezhadgashti H, Eris S, Pouroushaninia F, Ataeipour $\mathrm{Y}$, et al. Posttransplant diabetes mellitus in kidney allograft recipients at Shaheed Hasheminejad Hospital. Iranian J of Kidney Dis. 2008; 2(1).

28. Marghoob B, Ossareh S, Bayat R. Relationship Between Fetuin-A and Vascular or Valvular Calcification in Hemodialysis Patients. Iranian Journal of Kidney Diseases, 11:1, 16th International Congress of Nephrology, Dialysis, and Transplantation. 2017:107.

29. Rezapour M, Payani E, Taran M, Ghatari AR, Zadeh MK. Roles of triglyceride and phosphate in atherosclerosis of diabetic hemodialysis patients. Med J Islam Repub Iran. 2017;31:80.

30. Rezapour M, Khavaninzadeh M. Association between non-matured arterio-venus fistula and blood pressure in hemodialysis patients. Med J Islam Repub Iran. 2014;28:144.

31. Rezapour M, Zadeh MK, Sepehri MM, Alborzi M. Less primary fistula failure in hypertensive patients. J Human Hyperten. 2018 Mar 27:1.

32. Cronenwett JL, Johnston KW. Rutherford's Vascular Surgery E-Book. Elsevier Health Sciences; 2014 Mar 12. Section 2, Chapter 10: Zierler R.E., Sumner D. S., P. 133.

33. Byar D, Fiddian RV, Quereau M, Hobbs JT, Edwards EA. The fallacy of applying the Poiseuille equation to segmental arterial stenosis. Am Heart J. 1965 Aug 1;70(2):216-24

34. Fiddian RV, Byar D, Edwards EA. Factors affecting flow through a stenosed vessel. Archives of surgery. 1964 Jan 1;88(1):83-90.

35. Kindt GW, Youmans JR. The effect of stricture length on critical arterial stenosis. Surg Gynecol Obstetr. 1969 Apr;128(4):729.

36. May AG, Deweese JA, Rob CG. Hemodynamic effects of arterial stenosis. Surgery. 1963 Apr 1;53(4):513-24.

37. Ene-Iordache B, Cattaneo L, Remuzzi A, Dubini G. The anastomotic angle does change the disturbed flow patterns in end-to-side avf for haemodialysis. J Biomechanic. 2012 Jul 1;45:S127.

38. Ene-Iiordache B. Haemodynamics and vascular remodeling in vascular access: insights from numerical studies (Doctoral dissertation). Eindhoven University of Technology, Italy; 2015.

39. Ene-Iordache B, Cattaneo L, Dubini G, Remuzzi A. Effect of anastomosis angle on the localization of disturbed flow in 'side-toend'fistulae for haemodialysis access. Nephrol Dialysis Transplant. 2012 Jul 10;28(4):997-1005.

40. de Andrade Silva J, Karam-Filho J, Borges CC. Computational analysis of anastomotic angles by blood flow conditions in side-to-end radio-cephalic fistulae used in hemodialysis. J Biomedical Sci Engineer. 2015 Mar 17;8(03):131.

41. Grus T, Lambert L, Matecha J, Grusová G, Spacek M, Mlcek M. The ratio of diameters between the target artery and the bypass modifies hemodynamic parameters related to intimal hyperplasia in the distal end-to-side anastomosis. Physiol Res. 2016 Nov 1;65(6):901.

42. Hassan WA, Osman K, Kadir MR, Abdullah WA, Haron J, Ngali MZ. Effect of anastomosis angle on hemodynamic of side-to-end radiocephalic arteriovenous fistula (RCAVF). InAIP Conference Proceedings. 2012 Jun 29;1440(1): 665-670).

43. Van Canneyt K, Pourchez T, Eloot S, Guillame C, Bonnet A, Segers $\mathrm{P}$, Verdonck P. Hemodynamic impact of anastomosis size and angle in side-to-end arteriovenous fistulae: a computer analysis. J Vascular Access. 2010;11(1):52-8.

44. Hull JE, Balakin BV, Kellerman BM, Wrolstad DK. Computational fluid dynamic evaluation of the side-to-side anastomosis for arteriovenous fistula. J Vascular Surg. 2013 Jul 1;58(1):187-93.

45. Patel MS, Street T, Davies MG, Peden EK, Naoum JJ. Evaluating and treating venous outflow stenoses is necessary for the successful open surgical treatment of arteriovenous fistula aneurysms. J Vascular Surg. 2015 Feb 1;61(2):444-8.

46. Gnasso A, Carallo C, Irace C, De Franceschi MS, Mattioli PL, Motti C, Cortese C. Association between wall shear stress and flow-mediated vasodilation in healthy men. Atherosclerosis. 2001 May 1;156(1):1716. 